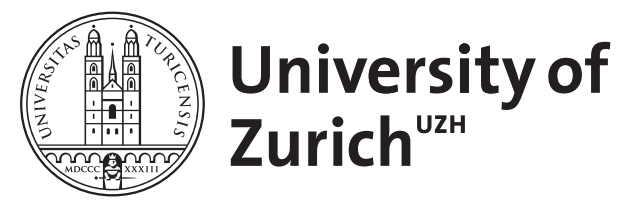

\title{
Hitzschlag mit Alpha-Koma
}

Gandjour, J ; Oehler, J ; Mohr, A ; Schellinger, P D

\begin{abstract}
Wir berichten über einen 41-jährigen komatösen Patienten, der einen schwer verlaufenden Hitzschlag mit einer Körperkerntemperatur von $41,5^{\circ} \mathrm{C}$ erlitt. Der klinische Verlauf wurde kompliziert durch eine akute systemische inflammatorische Reaktion und Multiorganversagen. Das EEG zeigte ein prognostisch ungünstiges Alpha-Koma ohne Reaktivität auf externe Stimuli. Der Patient erlangte das Bewusstsein wieder und wurde nach 16Tagen aus der intensivmedizinischen Behandlung entlassen. Im weiteren Verlauf entwickelte sich eine zerebrale Toxoplasmose, die mit einer Kombinationstherapie aus Sulfadiazin und Pyrimethamin behandelt wurde. Nach 65Tagen erfolgte eine Verlegung in eine Neurorehabilitationsklinik mit einem moderaten neurologischen Defizit
\end{abstract}

DOI: https://doi.org/10.1007/s00115-004-1715-6

Posted at the Zurich Open Repository and Archive, University of Zurich

ZORA URL: https://doi.org/10.5167/uzh-156352

Journal Article

Published Version

Originally published at:

Gandjour, J; Oehler, J; Mohr, A; Schellinger, P D (2005). Hitzschlag mit Alpha-Koma. Der Nervenarzt, 76(4):467-470.

DOI: https://doi.org/10.1007/s00115-004-1715-6 
Nervenarzt $2005 \cdot 76: 467-470$

DOI 10.1007/s00115-004-1715-6

Online publiziert: 1. Mai 2004

(c) Springer Medizin Verlag 2005

J. Gandjour ${ }^{1,3} \cdot$ J. Oehler ${ }^{1,4} \cdot$ A. Mohr ${ }^{2,5}$.P. D. Schellinger ${ }^{1,6}$

${ }^{1}$ Neurologische Klinik, Universität Heidelberg $\cdot{ }^{2}$ Abteilung Neuroradiologie, Universität Heidelberg $\cdot{ }^{3}$ Neurochirurgische Klinik, Universitätsspital Zürich

${ }^{4}$ Neurologische Klinik, Städtisches Klinikum Karlsruhe $\cdot{ }^{5}$ Abteilung Neuroradiologie, Universität Göttingen ${ }^{6} \mathrm{NINDS}$, NIH, Bethesda, MD, USA

\section{Hitzschlag mit Alpha-Koma}

\section{Ein Fallbericht}

W

ährend einer Hitzwelle kollabierte ein 41-jähriger Patient ohne Vorzeichen beim Ausführen geringer körperlicher Arbeit im Freien. Der hinzugerufene Notarzt fand einen dehydrierten, bewusstlosen Patienten (GCS: 3 ) mit engen lichtstarren Pupillen, ausgefallenen Hirnstammreflexen, erloschenen Muskeleigenreflexen und einer Körperkerntemperatur von $41,5^{\circ} \mathrm{C}$. Die Vitalparameter ergaben folgende Messwerte: Sauerstoffsättigung: 90\%, Puls: rhythmisch, 150/min, systolischer Blutdruck: 85 mmHg, Blutzucker: $218 \mathrm{mg} /$ dl. Vor Ort wurde unter Gabe von Thiopental ( $375 \mathrm{mg})$, Fentanyl (0,5 mg), Midazolam (10 mg) und Suxamethonium (100 mg) eine Intubationsnarkose eingeleitet. Thiopental wird nicht selten am Ereignisort prophylaktisch als hirndrucksenkendes Medikament verabreicht. Die Ursachen für die hohe Dosierung von Fentanyl und die Verabreichung von Suxamethonium bei einem Patienten mit einem GCS von 3 bleiben ungeklärt. Ungefähr $2 \mathrm{~h}$ nach Symptombeginn erfolgte die Aufnahme auf unsere Neurointensivstation. Die Körperkerntemperatur betrug $39,1^{\circ} \mathrm{C}$ und der neurologische Status war unter fortlaufender Analgosedierung mit Fentanyl und Midazolam unverändert zum Vorbefund.

\section{Laborparameter und Zusatzuntersuchungen}

\section{Relevante Laborparameter bei Aufnahme}

Arterielle Blutgasanalyse: metabolische Azidose, pH-Wert: 7,17; Blutalkoholspiegel und qualitativer Drogenschnelltest (Tria-
ge-Test, Nachweis von 8 Drogenklassen im Urin): nur Nachweis auch iatrogen verabreichter Substanzen: Barbiturate, Benzodiazepine und Opiate.

\section{Laborparameter innerhalb der ersten $48 \mathrm{~h}$}

Alle relevanten Laborparameter sind in - Tabelle 1 aufgelistet. Elektrolyte, Ammoniak, C-reaktives Protein und Schilddrüsenwerte lagen im Normbereich oder waren nur geringfügig erhöht, bzw. erniedrigt. Thiopental: unterhalb der Nachweisgrenze am 2. Behandlungstag; neuronenspezifische Enolase: bis zu 26,2 $\mu \mathrm{g} / \mathrm{l}$ am 3. Behandlungstag, im Verlauf rückläufig (Normwert $<17 \mu \mathrm{g} / \mathrm{l}$ ).

\section{Zusatzuntersuchungen innerhalb der ersten $48 \mathrm{~h}$}

Kraniale CT mit CT-Angiographie: regelrecht bis auf eine lateral des linken Hinterhorns lokalisierte Zyste (• Abb. 1); extraund intrakranielle Dopplersonographie: regelrecht bis auf allseits erhöhte Flussgeschwindigkeiten; EEG: Alpha-Koma ohne Reaktivität auf akustische und nozizeptive Reize bei komatösem, seit $29 \mathrm{~h}$ nicht analgosediertem Patienten (• Abb. 2); EKG: regelrecht bis auf Sinustachykardie; Thoraxröntgen und transthorakale Echokardiographie: regelrecht; Oberbauchsonographie: regelrecht bis auf Hepatomegalie.

\section{Zusatzuntersuchungen im Verlauf}

Kraniale MRT: regelrecht am 4. und 14. Behandlungstag bis auf eine wahrscheinlich neuroepitheliale Zyste, die sich vom linken lateralen Temporalhorn bis in das Zentrum semiovale erstreckt und den Ventrikel nach medial und kaudal verlagert, eine Kommunikation mit dem Ventrikelsystem ist nicht eindeutig nachweisbar, keine Kontrastmittelanreicherung; EEG-Verlaufskontrollen: beständige Befundverbesserung, am 15. Behandlungstag nur Grundrhythmusverlangsamung auf 6 bis $7 \mathrm{~Hz}$ bei wachem Patienten; NLG, EMG, SEP und AEP: regelrecht.

\section{Diagnose, Verlauf und Therapie}

Unser Patient erlitt einen Hitzschlag. Der Notarzt leitete frühzeitig eine intravenöse Flüssigkeitssubstitution und externe Kühlmaßnahmen ein, die Körperkerntemperatur wurde $3 \mathrm{~h}$ nach Symptombeginn auf normotherme Werte gesenkt. Im Verlauf kam es zu einer systemischen inflammatorischen Reaktion und Multiorganversagen

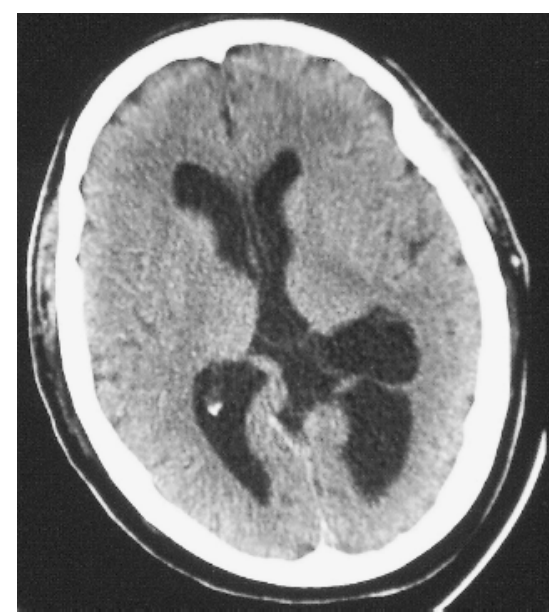

Abb. $1 \Delta$ Kraniale Nativ-CT, ca. 47 h nach Symptombeginn 


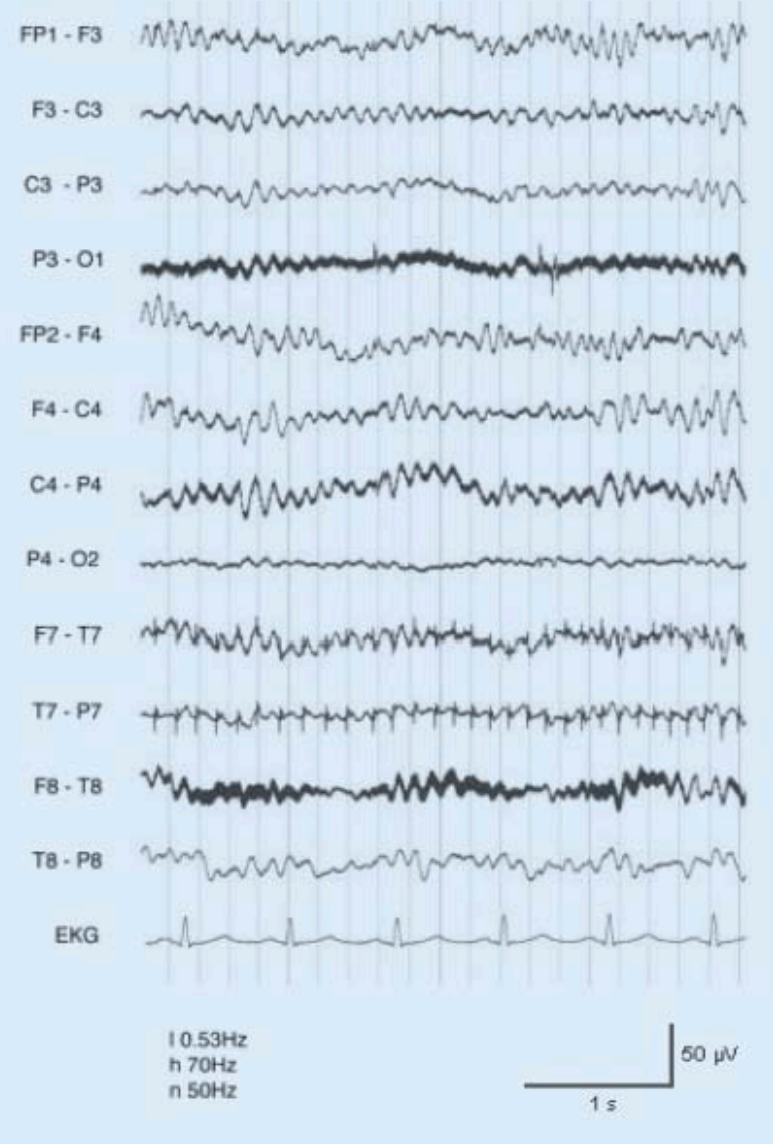

risiert durch akutes Auftreten ohne Prodromalerscheinungen, Bewusstseinsstörung, warme trockene Haut und Hyperthermie mit einer Körperkerntemperatur von über $40^{\circ} \mathrm{C}$. Häufig kommt es zu einer systemischen inflammatorischen Reaktion (SIRS) und Multiorganversagen [2]. Der klassische Hitzschlag resultiert aus extremer exogener Wärmeexposition bei verminderter Wärmeabgabe und betrifft häufig prämorbide ältere Menschen und Kleinkinder. Dem anstrengungsinduzierten Hitzschlag hingegen liegt ursächlich eine schwere körperliche Belastung mit endogener Hitzeproduktion zugrunde, gesunde Personen jeden Alters können hiervon betroffen sein [5]. Unser Patient hatte wahrscheinlich einen gemischt exogenen und endogenen Hitzschlag. Für einen exogenen Hitzschlag sprechen die fremdanamnestisch beschriebenen geringen Außenaktivitäten im Rahmen der Hitzewelle. Dagegen weisen das junge Lebensalter, fehlende internistische Vorerkrankungen und metabolische Störungen, wie Laktatazidose, Rhabdomyolyse und Nierenversagen auf einen endogenen Hitzschlag hin [5].

\section{Abb. $2<$ Alpha- Koma-EEG}

mit Verbrauchskoagulopathie, katecholaminpflichtigem Herz-Kreislauf-Versagen, Leberversagen, Rhabdomyolyse und nicht dialysepflichtigem Nierenversagen. Eine Infektfokussuche war negativ, Breitbandantibiotika wurden prophylaktisch verabreicht. Der Patient wurde am 7. Behandlungstag extubiert und war am 16. Behandlungstag wach, kontaktfähig und psychomotorisch verlangsamt. Ferner bestanden ein Strabismus convergens, linkes Auge adduziert ohne Angabe von Doppelbildern, sakkadierte Blickfolge nach oben, schwere Dysarthrie, Dysphagie und schwere Zeige-, Stand- und Gangataxie.

Es erfolgte eine Entlassung aus der intensivmedizinischen Behandlung und Verlegung in eine andere neurologische Klink. Dort wurde nach 11 Tagen und bei nahezu unverändertem neurologischen Status eine erneute kraniale MRT veranlasst. Hierbei kam es zur Darstellung von 2 ringförmig Kontrastmittel aufnehmenden, $13 \mathrm{~mm}$ messenden, links- und rechtsseitig parietal lokalisierten subkortikalen Läsionen. Eine am Folgetag durchgeführte Lumbalpunktion ergab Hinweise auf eine zerebrale Toxoplasmose. Eine Leukopenie be- stand zu keinem Zeitpunkt, der HIV-Status war negativ. Die Läsionen waren unter Behandlung mit Pyrimethamin, Sulfadiazin und Folinsäure rückläufig. Fünfundsechzig Tage nach Auftreten des Hitzschlages wurde der Patient mit einer ausgeprägten Dysarthrie, Dysphagie, einer diskreten Zeige- und moderaten Stand- und Gangataxie in eine Rehabilitationsklinik verlegt.

Eine retrospektive Beurteilung unserer Magnetresonanztomographien ergab keine Hinweise auf eine zu diesem Zeitpunkt vorhandene zerebrale Toxoplasmose. Unklar bleibt, welche neurologischen Symptome vor Auftreten des Hitzschlages bestanden. Der Patient berichtete den behandelnden Ärzten des auswärtigen Spitals über seit Jahren bestehende Augenbewegungsstörungen und mehrfach durchgeführte Lumbalpunktionen. Die Akten waren dem auswärtigen Spital jedoch nicht zugänglich.

\section{Diskussion}

\section{Differenzialdiagnose Hitzschlag}

Der Hitzschlag ist ein intensivmedizinischer Notfall und in der Regel charakte-

\section{Neurostatus und Prognose}

Eine Beobachtungsstudie untersuchte 87 Patienten, die einen Hitzschlag während einer Pilgerfahrt erlitten [10]: 30\% waren tief komatös, hatten miotische Pupillen und erloschene Hirnstamm- und Muskeleigenreflexe. Die Mortalitätsrate lag bei ca. $12 \%$. Eine andere Beobachtungsstudie untersuchte 58 Patienten mit klassischem Hitzschlag, die während einer Hitzwelle intensivmedizinisch behandelt wurden [4]: 75\% aller Patienten waren bei Aufnahme komatös, $16 \%$ hatten epileptische Anfälle. In der Elektroenzephalographie fanden sich keine fokalen Veränderungen; ergänzende Daten, beispielsweise über die Schwere von generalisierten Veränderungen, wurden nicht veröffentlicht. Die kraniale Bildgebung war in 35\% aller Fälle unauffällig. Drei Patienten hatten unspezifische Akutveränderungen, hierunter ein $\mathrm{Pa}$ tient mit zerebralem Ödem. Während des stationären Aufenthaltes verstarben $21 \%$ aller Patienten, weitere $28 \%$ innerhalb des darauf folgenden Jahres. Bei Entlassung waren $24 \%$ aller überlebenden Patienten fokal-neurologisch unauffällig, $43 \%$ wie- 
sen geringe und 33\% moderate bis schwere Beeinträchtigungen auf. Einige Patienten entwickeln eine zerebelläre Atrophie mehrere Wochen nach Auftreten eines Hitzschlages, neurologische und neuroradiologische Verlaufskontrollen werden empfohlen [1].

\section{Therapie}

Rasche Kühlung und Unterstützung der Vitalfunktionen sind die entscheidenden Therapiemaßnahmen [2]. Kontrollierte Studien über die effektivsten Kühlmethoden wurden nicht veröffentlicht. Im Allgemeinen werden evaporative Kühlmethoden bevorzugt [5]: Die Körperoberfläche wird mit Wasser benetzt und der Luftzug über dem Patienten erhöht. Diese Maßnahmen können durch Blasenspülung oder intravenöse Kühlkatheter ergänzt werden. Bei der Immersionsmethode wird in einer Wanne mit Kaltwasser gekühlt, die Basisund Notversorgung der Patienten aber erheblich erschwert. Dantrolen war in einer doppelblind kontrollierten Studie unwirksam [3]. Die Anwendung von Antipyretika wurde nicht evaluiert, ist aber bei Auftreten eines SIRS vorstellbar [2]. Andere Autoren sehen keine Indikation für die Verabreichung antipyretischer Substanzen, weil der Hitzschlag primär nicht auf Veränderungen des hypothalamischen Temperatursollwerts beruht [6]. Auch der Nutzen anderer entzündungshemmender und -modulierender Substanzen bleibt unklar. Eine Vielzahl unterschiedlicher Substanzen wird im Rahmen septischer Krankheitsbilder eingesetzt wie aktiviertes Protein C [8] und Antithrombin III [9]. Auch unser Patient entwickelte ein SIRS, und wir vermuten, dass es im Rahmen einer sekundären Immunschwäche zur Reaktivierung einer latenten Toxoplasmoseinfektion kam. Unseres Wissens wurden in der Literatur keine Fälle mit Exazerbation einer Toxoplasmose nach Hitzschlag beschrieben.

\section{EEG}

Die Anwendung kontinuierlicher oder serieller EEG und Testung der Reaktivität auf externe Stimuli ermöglicht eine prognostische Beurteilung komatöser Neurointensivpatienten [7]. Bei unserem Patienten wurde ein Alpha-Koma abgeleitet ohne Reak-

Nervenarzt $2005 \cdot 76: 467-470$

DOI 10.1007/s00115-004-1715-6

() Springer Medizin Verlag 2005

\section{J. Gandjour · J. Oehler · A. Mohr · P. D. Schellinger}

\section{Hitzschlag mit Alpha-Koma. Ein Fallbericht}

Zusammenfassung

Wir berichten über einen 41-jährigen komatösen Patienten, der einen schwer verlaufenden Hitzschlag mit einer Körperkerntemperatur von $41,5^{\circ} \mathrm{C}$ erlitt. Der klinische Verlauf wurde kompliziert durch eine akute systemische inflammatorische Reaktion und Multiorganversagen. Das EEG zeigte ein prognostisch ungünstiges Alpha-Koma ohne Reaktivität auf externe Stimuli. Der Patient erlangte das Bewusstsein wieder und wurde nach 16 Tagen aus der intensivmedizinischen Behandlung entlassen. Im weiteren Verlauf entwickelte sich eine zerebrale Toxoplasmose, die mit einer Kombinationstherapie aus Sulfadiazin und Pyrimethamin behandelt wurde. Nach 65 Tagen erfolgte eine Verlegung in eine Neurorehabilitationsklinik mit einem moderaten neurologischen Defizit.

\section{Schlüsselwörter}

Alpha-Koma · Hitzschlag · Hyperthermie · Multiorganversagen $\cdot$ SIRS

\section{Heat stroke with alpha coma. A case report}

\section{Summary}

We report the case of a near-fatal heat stroke in a 41-year-old patient. The comatose patient had a body core temperature of $41.5^{\circ} \mathrm{C}$. The clinical course was complicated by systemic inflammatory response syndrome and multiorgan failure. The EEG showed an alpha coma that did not react to external stimuli and, in general, has a poor prognosis. The patient regained consciousness and was discharged from our intensive care unit after 16 days. In the fur- ther course cerebral toxoplasmosis developed which was treated with a combination therapy of sulfadiazine and pyrimethamine. The patient was transferred to a neurorehabilitation clinic with a moderate neurological deficit 65 days after heat stroke onset.

Keywords

Alpha coma · Heat stroke · Hyperthermia . Multiorgan failure $\cdot$ SIRS 
Tabelle 1

Relevante Laborparameter innerhalb der ersten $48 \mathrm{~h}$

\begin{tabular}{llll}
\hline Parameter & Wert & Normwert \\
\hline Kreatinin & $3,35 \uparrow$ & $<1,30 \mathrm{mg} / \mathrm{dl}$ \\
\hline Harnstoff & $138 \uparrow$ & $<45 \mathrm{mg} / \mathrm{dl}$ \\
\hline CK & $1.535 \uparrow$ & $<80 \mathrm{U} / \mathrm{l}$ \\
\hline cTNI & $22,0 \uparrow$ & $<0,6 \mu \mathrm{g} /$ \\
\hline cTNT & $1,16 \uparrow$ & $<0,1 \mu \mathrm{g} / \mathrm{l}$ \\
\hline LDH & $4.328 \uparrow$ & $120-240 \mathrm{U} / \mathrm{l}$ \\
\hline GOT/ASAT & $1.456 \uparrow$ & $<18 \mathrm{U} / \mathrm{l}$ \\
\hline GPT/ALT & $1.966 \uparrow$ & $<24 \mathrm{U} / \mathrm{l}$ \\
\hline GGT & $1.259 \uparrow$ & $3-28 \mathrm{U} / \mathrm{l}$ \\
\hline Bilirubin & $2,5 \uparrow$ & $<0,1 \mathrm{mg} / \mathrm{dl}$ \\
gesamt & & \\
\hline Bilirubin & $1,5 \uparrow$ & $<0,3 \mathrm{mg} / \mathrm{dl}$ \\
direkt & & \\
\hline Hämoglobin & $10,3 \downarrow$ & $13,0-17,0 \mathrm{~g} / \mathrm{dl}$ \\
\hline Erythrozyten & $3,4 \downarrow$ & $4,3-6,1 / \mathrm{pl}$ \\
\hline Thrombo- & $37 \downarrow$ & $150-440 / \mathrm{nl}$ \\
zyten & & \\
\hline Leukozyten & $11,2 \uparrow$ & $4-10 / \mathrm{nl}$ \\
\hline Quick & $28 \downarrow$ & $70-125 \%$ \\
\hline INR & $2,4 \uparrow$ & $<1,2$ \\
\hline PTT & $48,9 \uparrow$ & $<35 \mathrm{~s}$ \\
\hline Fibrinogen & $1,59 \downarrow$ & $1,7-4,5 \mathrm{~g} / \mathrm{l}$ \\
\hline AT III & $58 \downarrow$ & $80-120 \%$ \\
\hline D-Dimere & $3,03 \uparrow$ & $<0,15 \mathrm{mg} / \mathrm{l}$ \\
\hline & & \\
\hline
\end{tabular}

Es werden die jeweils niedrigsten $(\downarrow)$ bzw. höchsten Werte ( $\uparrow$ ) angegeben.

tivität auf akustische und nozizeptive Reize. Der Patient erhielt seit $29 \mathrm{~h}$ keine Analgosedativa; der Thiopentalspiegel lag unterhalb der Nachweisgrenze. Ein medikamentös induziertes Alpha-Koma kann dennoch nicht ausgeschlossen werden. Die kranialen MRT, SEP und AEP ergaben keinen Hinweis auf eine subkortikale (subhemisphärielle) Ursache. Am 15. Behandlungstag waren nur geringe Allgemeinveränderungen nachweisbar. Eine Metaanalyse untersuchte 335 in der Literatur beschriebene Patienten mit Alpha-Koma [7]. Zu den Ursachen zählten Hypoxien mit und ohne kardiopulmonale Reanimation, Schlaganfälle und Intoxikationen. Mit Ausnahme von Intoxikationen wies das Alpha-Koma eine schlechte Prognose auf. Die Gesamtmortalität lag bei $76 \%$, Patienten mit erhaltener Reaktivität hatten eine bessere Prognose.

\section{Fazit für die Praxis}

Zusammenfassend handelt es sich beim Hitzschlag um ein potenziell lebensbedrohliches Krankheitsbild, das bei frühzeitiger Erkennung und Therapie einen günstigen Verlauf mit kompletter Remission nehmen kann. Durch eine exakte Erhebung von Anamnese und Messung der Körperkerntemperatur kann bereits am Ereignisort differenzialdiagnostisch eine Abgrenzung zu anderen Bewusstseinsstörungen erfolgen. Es ist davon auszugehen, dass aufgrund der globalen Erwärmung auch in unseren Breitengraden die Inzidenz des Hitzschlages zunehmen wird.

\section{Korrespondierender Autor Dr. J. Gandjour}

Neurointensivstation Neurochirurgische Klinik, Universitätsspital Zürich,

Frauenklinikstrasse 10, 8091 Zürich, Schweiz E-Mail: joubin.gandjour@usz.ch

Interessenkonflikt: Der korrespondierende Autor versichert, dass keine Verbindungen mit einer Firma, deren Produkt in dem Artikel genannt ist, oder einer Firma, die ein Konkurrenzprodukt vertreibt, bestehen.

\section{Literatur}

1. Albukrek D, Bakon M, Moran DS et al. (1997) Heatstroke-induced cerebellar atrophy: clinical course, CT and MRI findings. Neuroradiology 39:195-197

2. Bouchama A, Knochel JP (2002) Heat stroke. N Engl J Med 346:1978-1988

3. Bouchama A, Cafege A, Devol EB et al. (1991) Ineffectiveness of dantrolene sodium in the treatment of heatstroke. Crit Care Med 19:176-180

4. Dematte JE, O'Mara K, Buescher J et al. (1998) Nearfatal heat stroke during the 1995 heat wave in Chicago. Ann Intern Med 129:173-181

5. Dunker M, Rehm M, Thiel M et al. (2001) Exertional heatstroke. Lethal multi-organ failure due to hyperthermia in a 23-year-old sportsman. Anaesthesist 50:500-505

6. Hett HA, Brechtelsbauer DA (1998) Heat-related illness. Plan ahead to protect your patients. Postgrad Med 103:107-120

7. Kaplan PW, Genoud D, Ho TW et al. (1999) Etiology, neurologic correlations, and prognosis in alpha coma. Clin Neurophysiol 110:205-213

8. PROWESS study group (2001) Efficacy and safety of recombinant human activated protein $\mathrm{C}$ for severe sepsis. N Engl J Med 344:699-709

9. Rublee D, Opal SM, Schramm W et al. (2002). Quality of life effects of antithrombin III in sepsis survivors: results from the KyberSept trial [ISRCTN22931023]. Crit Care 6:349-356

10. Yaqub B, Al Deeb S (1998) Heat strokes: aetiopathogenesis, neurological characteristics, treatment and outcome. J Neurol Sci 156:144-151 www.jobcenter-medizin.de

Neuer Online-Stellenmarkt für Ärzte

Die Springer-Medizin-Verlage haben eine neue, große Stellenbörse für Mediziner gegründet. Unter www.jobcenter-medizin.de ist diese seit März 2005 online.

Das Angebot richtet sich sowohl an Ärzte, die einen Job suchen als auch an Kliniken, Praxen und Firmen, die Ärzte einstellen möchten.

Die Vorteile für Stellenanbieter:

— kostengünstiger als Printmedien

— unkomplizierte Abwicklung

- sofortiges Erscheinen der Anzeige

- wahlweise: selbstständige Eingabe von

Anzeigen oder Eingabe durch Mitarbeiter des Portals nach bestimmten Gestaltungswünschen

— reichweitenstarke Plattform

Die Vorteile für Stellensuchende:

- nutzerfreundliches, übersichtliches Design

— schnelle, komfortable, präzise Suchfunktionen

- Erstellung persönlicher Suchprofile und Benachrichtigung über aktuelle, offene Angebote per Mail

- fachspezifische Stellensuche

Der Bekanntheitsgrad der neuen Plattform und ihre Reichweite werden durch die Zahl der angeschlossenen Verlage garantiert. Die Springer-Medizin-Verlage sind im deutschsprachigen Raum einer der wichtigsten Partner für Ärzte und medizinische Berufsgruppen, Fachgesellschaften, Berufsverbände sowie Institutionen im Gesundheitswesen. Allein die Springer-Portale „Ärzte Zeitung online“, "Medizin-Online" und "Lifeline" erzielen zusammen monatlich mehr als 5 Millionen Seitenabrufe. Jobcenter-medizin.de wird integraler Bestandteil auf allen SpringerOnline-Portalen sein.

\section{Quelle: www.jobcenter-medizin.de,} info@jobcenter-medizin.de 